Bulletin of the Section of Logic

Volume 50/3 (2021), pp. 299-324

https://doi.org/10.18778/0138-0680.2021.11

Akbar Paad

\title{
TENSE OPERATORS ON BL-ALGEBRAS AND THEIR APPLICATIONS
}

\begin{abstract}
In this paper, the notions of tense operators and tense filters in $B L$-algebras are introduced and several characterizations of them are obtained. Also, the relation among tense $B L$-algebras, tense $M V$-algebras and tense Boolean algebras are investigated. Moreover, it is shown that the set of all tense filters of a $B L$-algebra is complete sublattice of $F(L)$ of all filters of $B L$-algebra $L$. Also, maximal tense filters and simple tense $B L$-algebras and the relation between them are studied. Finally, the notions of tense congruence relations in tense $B L$-algebras and strict tense $B L$-algebras are introduced and an one-to-one correspondence between tense filters and tense congruences relations induced by tense filters are provided.
\end{abstract}

Keywords: (simple) tense $B L$-algebra, tense operators, tense filter, tense congruence.

2000 Mathematical Subject Classification: 06E99, 03G25.

\section{Introduction}

$B L$-algebras are the algebraic structures for Hájek Basic logic [8], in order to investigate many valued logic by algebraic means. His motivations for introducing $B L$-algebras were of two kinds. The first one was providing an algebraic counterpart of a propositional logic, called Basic Logic, which embodies a fragment common to some of the most important many-valued logics, namely Lukasiewicz Logic, Gödel Logic and Product Logic. This

Presented by: Janusz Ciuciura

Received: December 9, 2019

Published online: May 28, 2021

(C) Copyright by Author(s), Łódź 2021

(C) Copyright for this edition by Uniwersytet Łódzki, Łódź 2021 
Basic Logic ( $B L$ for short) is proposed as "the most general" many-valued logic with truth values in $[0,1]$ and $B L$-algebras are the corresponding Lindenbaum-Tarski algebras. The second one was to provide an algebraic mean for the study of continuous t-norms (or triangular norms) on $[0,1]$. Most familiar example of a $B L$-algebra is the unit interval $[0,1]$ endowed with the structure induced by a continuous t-norm. In 1958, Chang introduced the concept of an $M V$-algebra which is one of the most classes of $B L$-algebras. $M V$-algebras, Gödel algebras and product algebras are the most known classes of $B L$-algebras. Hájek in [8], introduced the notions of filters and prime filters in $B L$-algebra and by using the prime filters of $B L$-algebras, he proved the completeness of basic logic $B L$. Filter theory play an important rule in studying these algebras. From logical point of view, various filter correspond to various set of provable formulas.

Study of tense operators was originated in 1980's, see e.g. a compendium [2]. The classical tense logic is a logical system obtained from the bivalent logic by adding the tense operators $G$ (it is always going to be the case that) and $H$ (it has always been the case that). Starting with other logical systems (intuitionistic calculus, many-valued logics etc.) and adding appropriate tense operators we arrive to new tense logics. Two other operators $F$ and $P$ are usually defined via $G$ and $H$ by $F(x)=\neg G(\neg x)$ and $P(x)=\neg H(\neg x)$, where $\neg x$ denotes negation of the proposition $x$. So, $G$ and $H$ can be recognized as tense for all quantifiers and $P$ and $F$ as tense existential quantifiers. Recall that for a classical propositional calculus represented by means of a Boolean algebra $B=(B, \vee, \wedge, \neg, 0,1)$, tense operators were axiomatized in [2] by the following axioms:

(B1) $G(1)=1, H(1)=1$,

(B2) $G(x \wedge y)=G(x) \wedge G(y), H(x \vee y)=H(x) \vee H(y)$,

(B3) $\neg G \neg H(x) \leq x, \neg H \neg G(x) \leq x$.

For Boolean algebras, the axiom (B3) is equivalent to

(B3') $G(x) \vee y=x \vee H(y)$.

To introduce tense operators in non-classical logics, some more axioms must be added on $G$ and $H$ to express connections with additional operations or logical connectives. Tense operators have been studied by different authors for various classes of algebras. For example, tense operators on Basic algebras and effect algebras, on $M V$-algebras and Lukasiewicz-Moisil algebras 
and on intuitionistic logic (corresponding to Heyting algebras) were studied by Botur et al. [1], Diaconescu et al. [5] and Chajda [3], respectively. This motivated us to introduce tense operators on the structure of $B L$-algebras as an extension of the tense $M V$-algebras and because there was an negation on $B L$-algebras, the operators $F$ and $P$ were introduced as similar to tense operators on $M V$-algebras with two additional conditions. For other interesting algebras the reader is referred to $[4,7,6,9]$. This paper is organized as follows:

Section 2 contains some fundamental definitions and results. In Section 3 we introduce the notion of tense operators on $B L$-algebras and we study relation among tense $B L$-algebras, tense $M V$-algebras and tense Boolean algebras. In Section 4 we introduce the notion of tense filters on $B L$ algebras and we prove that the set of all tense filters of a $B L$-algebra is complete sublattice of $F(L)$ of all filters of $B L$-algebra $L$. Also, we study maximal tense filters and simple tense $B L$-algebras and the relation between them. In Section 5 we introduce the notions of tense congruence in tense $B L$-algebras and strict tense $B L$-algebras and we give some related results.

\section{Preliminaries}

In this section, we give some fundamental definitions and results. For more details, refer to the references.

Definition 2.1. [8] A $B L$-algebra is an algebra $(L, \vee, \wedge, \odot, \rightarrow, 0,1)$ of type $(2,2,2,2,0,0)$ such that

$(B L 1)(L, \vee, \wedge, 0,1)$ is a bounded lattice,

$(B L 2)(L, \odot, 1)$ is a commutative monoid,

$(B L 3) z \leq x \rightarrow y$ if and only if $x \odot z \leq y$,

$(B L 4) x \wedge y=x \odot(x \rightarrow y)$,

$(B L 5)(x \rightarrow y) \vee(y \rightarrow x)=1$, for all $x, y, z \in L$.

A $B L$-algebra $L$ is called a Gödel algebra, if $x^{2}=x \odot x=x$, for all $x \in L$ and a $B L$-algebra $L$ is called an $M V$-algebra, if $\left(x^{-}\right)^{-}=x$, for all $x \in L$, where $x^{-}=x \rightarrow 0$. A $B L$-algebra $L$ is Boolean algebra if and only if $x^{2}=x$ and $\left(x^{-}\right)^{-}=x$, for all $x \in L$. 
Proposition 2.2. $[11,12]$ In any $B L$-algebra $L$ the following hold:

(BL6) $x \leq y$ if and only if $x \rightarrow y=1$,

$(B L 7) x \leq x^{--}$and $x \rightarrow(y \rightarrow z)=y \rightarrow(x \rightarrow z)$,

(BL8) $x \leq y$ implies $x \odot z \leq y \odot z, y \rightarrow z \leq x \rightarrow z$ and $z \rightarrow x \leq z \rightarrow y$,

$(B L 9) y \rightarrow x \leq(z \rightarrow y) \rightarrow(z \rightarrow x)$,

$(B L 10) x \rightarrow(y \rightarrow z)=x \odot y \rightarrow z$,

$(B L 11) x \odot y=0$ if and only if $x \leq y^{-}$,

$(B L 12) x^{---}=x^{-}, x \leq y \rightarrow x$ and $x \odot x^{-}=0$,

$(B L 13) x \rightarrow \underset{i \in I}{\wedge} y_{i}=\wedge_{i \in I}\left(x \rightarrow y_{i}\right)$,

$(B L 14)(x \wedge y)^{--}=x^{--} \wedge y^{--},(x \rightarrow y)^{--}=x^{--} \rightarrow y^{--}$and $(x \odot y)^{--}=$ $x^{--} \odot y^{--}$, for all $x, y, z, y_{i} \in L$.

Definition $2.3([11,12])$. Let $L$ be a $B L$-algebra and $F$ be a nonempty subset of $L$. Then

(i) $F$ is called a filter of $L$ if $x \odot y \in F$, for any $x, y \in F$ and if $x \in F$ and $x \leq y$ then $y \in F$, for all $x, y \in L$.

(ii) $F$ is called a maximal filter of $L$ if it is a proper filter and is not properly contained in any other proper filter of $L$.

(iii) $L$ is called a simple $B L$-algebra if $L$ is non-trivial and $\{1\}$ is its only proper filter.

TheORem $2.4([8])$. Let $F$ be a filter of BL-algebra $L$. Then the binary relation $\equiv_{F}$ on $L$ which is defined by

$$
x \equiv_{F} y \text { if and only if } x \rightarrow y \in F \quad \text { and } y \rightarrow x \in F
$$

is a congruence relation on L.(Filters of $L$ and congruence relations $\equiv_{F}$ on $L$ are in one-to-one correspondence.) Define $\cdot, \rightarrow, \sqcup, \sqcap$ on $\frac{L}{F}$, the set of all congruence classes of $L$, as follows:

$[x] \cdot[y]=[x \odot y],[x] \rightarrow[y]=[x \rightarrow y],[x] \sqcup[y]=[x \vee y],[x] \sqcap[y]=[x \wedge y]$.

Then $\left(\frac{L}{F}, \cdot, \rightarrow, \sqcup, \sqcap,[0],[1]\right)$ is a $B L$-algebra which is called quotient $B L$ algebra with respect to $F$. 
Definition 2.5. An $M V$-algebra is an algebra $(L, \oplus, \neg, 0,1)$ of type $(2,1,0)$ satisfying the following axioms for any $x, y, z \in L$ :

$(M V 1) x \oplus y=y \oplus x$,

$(M V 2) x \oplus(y \oplus z)=(x \oplus y) \oplus z$,

$(M V 3) x \oplus 0=x$,

$(M V 4) \neg \neg x=x$,

$(M V 5) x \oplus 1=1$, where $1:=\neg 0$,

$(M V 6) \neg(\neg x \oplus y) \oplus y=\neg(\neg y \oplus x) \oplus x$.

In any $M V$-algebra $L$ we can introduce the new operations $\odot, \vee, \wedge$ and $\rightarrow$ for any $x, y \in L$ as follow:

$x \odot y=\left(x^{-} \oplus y^{-}\right)^{-}, x \vee y=x \oplus(\neg x \odot y)=y \oplus(\neg y \odot x), x \wedge y=$ $x \odot(\neg x \oplus y)=y \odot(\neg y \oplus x)$ and $x \rightarrow y=\neg x \oplus y$.

Definition 2.6. [5] Let $(L, \oplus, \neg, 0,1)$ be an $M V$-algebra and $G, H: L \rightarrow$ $L$, be two unary operations on $L$. Then the structure $(L ; G, H)$ is called a tense $M V$-algebra if it satisfies in the following conditions for any $x, y \in L$ : (A0) $G(1)=1, H(1)=1$,

$(A 1) G(x \rightarrow y) \leq G(x) \rightarrow G(y), H(x \rightarrow y) \leq H(x) \rightarrow H(y)$,

(A2) $G(x) \oplus G(y) \leq G(x \oplus y), H(x) \oplus H(y) \leq H(x \oplus y)$,

(A3) $G(x \oplus x) \leq G(x) \oplus G(x), H(x \oplus x) \leq H(x) \oplus H(x)$,

(A4) $F(x) \oplus F(x) \leq F(x \oplus x), P(x) \oplus P(x) \leq P(x \oplus x)$,

(A5) $x \leq G P(x), x \leq H F(x)$, where $F$ and $P$ are the unary operations of $L$ defined by $F(x)=(\neg G(\neg x)), P(x)=(\neg H(\neg x))$.

\section{Tense Operators on $B L$-algebras}

In this section, we introduce the notion of tense operators on $B L$-algebras and we give some related results. 
Definition 3.1. Let $(L, \vee, \wedge, \rightarrow, \odot, 0,1)$ be a $B L$-algebra and $G, H: L \rightarrow$ $L$ be two unary operations on $L$. The structure $(L ; G, H)$ is called a tense $B L$-algebra if the following conditions hold:

$(T B L 0) G(1)=1, H(1)=1$.

$(T B L 1) G(x \rightarrow y) \leq G(x) \rightarrow G(y), H(x \rightarrow y) \leq H(x) \rightarrow H(y)$.

(TBL2) $x \leq G P(x), x \leq H F(x)$, where $F$ and $P$ are two unary operations of $L$ defined by $F(x)=\left(G\left(x^{-}\right)\right)^{-}$and $P(x)=\left(H\left(x^{-}\right)\right)^{-}$, with additional conditions $\left(G\left(x^{--}\right)\right)^{--}=G(x)$ and $\left(H\left(x^{--}\right)\right)^{--}=H(x)$, for all $x, y \in L$.

Note that by additional conditions in Definition 3.1, we conclude that $\left(F\left(x^{-}\right)\right)^{-}=\left(G\left(\left(x^{-}\right)^{-}\right)^{-}\right)^{-}=\left(G\left(x^{--}\right)\right)^{--}=G(x)$ and $\left(P\left(x^{-}\right)\right)^{-}=$ $\left(H\left(\left(x^{-}\right)^{-}\right)^{-}\right)^{-}=\left(H\left(x^{--}\right)\right)^{--}=H(x)$. Hence $F$ and $G, P$ and $H$ are in some sense equivalent.

Example 3.2. [10] Let $L=\{0, a, b, 1\}$, where $0<a<b<1$ and $x \wedge y=$ $\min \{x, y\}, x \vee y=\max \{x, y\}$ and operations $\odot$ and $\rightarrow$ are defined as the following tables:

Table 1

\begin{tabular}{|c|c|c|c|c|}
\hline$\odot$ & 0 & $a$ & $b$ & 1 \\
\hline 0 & 0 & 0 & 0 & 0 \\
$a$ & 0 & 0 & 0 & $a$ \\
$b$ & 0 & 0 & $a$ & $b$ \\
1 & 0 & $a$ & $b$ & 1 \\
\hline
\end{tabular}

Table 2

\begin{tabular}{|l|l|l|l|l|}
\hline$\rightarrow$ & 0 & $a$ & $b$ & 1 \\
\hline 0 & 1 & 1 & 1 & 1 \\
$a$ & $b$ & 1 & 1 & 1 \\
$b$ & $a$ & $b$ & 1 & 1 \\
1 & 0 & $a$ & $b$ & 1 \\
\hline
\end{tabular}

Then $(L, \vee, \wedge, \odot, \rightarrow, 0,1)$ is a $B L$-algebra. We define the operations $G=H$ on $L$ as $G(0)=0, G(a)=a, G(b)=b, G(1)=1$. It is not difficult to check that $G$ and $H$ are tense operators on $L$ and so $(L ; G, H)$ is a tense $B L$-algebra.

Example 3.3. Every tense $M V$-algebra is a tense $B L$-algebra.

Recall that a frame is a pair $(X, R)$, where $X$ is a nonempty set and $R$ is a binary relation on $X[2]$. The notion of frame allows us to construct the second example of tense $B L$-algebra. Also, we mention that if $L$ is a $B L$-algebra and $X$ a set, then $L^{X}$ the set of all mappings from $X$ into $L$, together with the operations is a $B L$-algebra, 
- $(f \vee g)(x)=f(x) \vee g(x)$,

- $(f \wedge g)(x)=f(x) \wedge g(x)$,

- $(f \rightarrow g)(x)=f(x) \rightarrow g(x)$,

- $f(x \odot y)=f(x) \odot f(y), 0(x)=0,1(x)=1$.

Now, we define $L_{2}^{X}$ as follow:

$$
L_{2}^{X}=\left\{f \in L^{X} \mid f^{--}(x)=f(x), \text { for any } x \in X\right\}
$$

it is clear by $(B L 14), L_{2}^{X}$ is a sub $B L$-algebra of $L^{X}$.

Lemma 3.4. Let $L$ be a $B L$-algebra and $a_{i}, b_{i} \in L$, for any $i \in I$. Then

$$
\bigwedge_{i \in I}\left(a_{i} \rightarrow b_{i}\right) \odot \bigwedge_{i \in I} a_{i} \leq \bigwedge_{i \in I} b_{i}
$$

(whenever the arbitrary meets exist.)

Proof: Let $a_{i}, b_{i} \in L$, for any $i \in I$. Then by (BL13),

$$
\bigwedge_{i \in I} a_{i} \rightarrow \bigwedge_{i \in I} b_{i}=\bigwedge_{i \in I}\left(\bigwedge_{i \in I} a_{i} \rightarrow b_{i}\right)
$$

Now, since $\bigwedge_{i \in I} a_{i} \leq a_{i}$, for any $i \in I$, by $(B L 8)$, we get that $a_{i} \rightarrow b_{i} \leq$ $\bigwedge_{i \in I} a_{i} \rightarrow b_{i}$, for any $i \in I$ and so $a_{i} \rightarrow b_{i} \leq \bigwedge_{i \in I}\left(\bigwedge_{i \in I} a_{i} \rightarrow b_{i}\right)$. Hence,

$$
\begin{aligned}
\bigwedge_{i \in I}\left(a_{i} \rightarrow b_{i}\right) & \leq \bigwedge_{i \in I}\left(\bigwedge_{i \in I} a_{i} \rightarrow b_{i}\right) \\
& =\bigwedge_{i \in I} a_{i} \rightarrow \bigwedge_{i \in I} b_{i}
\end{aligned}
$$

Hence, by $(B L 3)$, we conclude that

$$
\bigwedge_{i \in I}\left(a_{i} \rightarrow b_{i}\right) \odot \bigwedge_{i \in I} a_{i} \leq \bigwedge_{i \in I} b_{i} .
$$


TheOREM 3.5. Let $L$ be a complete $B L$-algebra, $(X, R)$ be a frame with $R$ reflexive, $G^{*}$ and $H^{*}$ the unary operations on $B L$-algebra $L_{2}^{X}$ defined by

$$
\begin{aligned}
G^{*}(f)(x) & =\bigwedge\{f(y) \mid y \in X, x R y\} \\
H^{*}(f)(x) & =\bigwedge\{f(y) \mid y \in X, y R x\}
\end{aligned}
$$

for all $f \in L_{2}^{X}$ and $x \in X$. Then $\left(L_{2}^{X}, G^{*}, H^{*}\right)$ is a tense BL-algebra.

Proof: Let $x \in X$. Then

$$
\begin{aligned}
G^{*}(1)(x) & =\bigwedge\{1(y) \mid y \in X, x R y\} \\
& =\bigwedge\{1 \mid y \in X, x R y\} \\
& =1 .
\end{aligned}
$$

Similarly, $H^{*}(1)(x)=1$. For $f, g \in L_{2}^{X}$ and $x \in X$, we have

$$
\begin{aligned}
G^{*}(f \rightarrow g)(x) \odot G^{*}(f)(x)= & \bigwedge\{(f \rightarrow g)(y) \mid y \in X, x R y\} \\
& \odot \bigwedge\{f(y) \mid y \in X, x R y\} \\
= & \bigwedge\{f(y) \rightarrow g(y) \mid y \in X, x R y\} \\
& \odot \bigwedge\{f(y) \mid y \in X, x R y\} \\
\leq & \bigwedge\{g(y) \mid y \in X, x R y\}, \text { By Lemma } 3.4 \\
= & G^{*}(y)(x)
\end{aligned}
$$

and so by $(B L 3)$, we conclude that $G^{*}(f \rightarrow g)(x) \leq G^{*}(f)(x) \rightarrow G^{*}(g)(x)$. Hence, $G^{*}(f \rightarrow g) \leq G^{*}(f) \rightarrow G^{*}(g)$. Similarly, $H^{*}(f \rightarrow g) \leq H^{*}(f) \rightarrow$ $H^{*}(g)$. Moreover, for $f \in L_{2}^{X}$ and $x \in X$, we have

$$
\begin{aligned}
G^{*} P^{*}(f)(x) & =G^{*}\left(\left(H\left(f^{-}\right)\right)^{-}(x)\right) \\
& =\bigwedge\left\{\left(H\left(f^{-}\right)\right)^{-}(y) \mid x R y, y \in X\right\} .
\end{aligned}
$$

Now, by $(B L 7)$, we get that 


$$
\begin{aligned}
\left(H\left(f^{-}\right)(y)\right)^{-} & =\left(\bigwedge\left\{f^{-}(z) \mid z R y\right\}\right)^{-} \\
& =\bigvee\left\{f^{--}(z) \mid z R y\right\} \\
& =\bigvee\{f(z) \mid z R y\} .
\end{aligned}
$$

Since $x R y$, we get that $\bigvee\{f(z) \mid z R y\} \geq f(x)$. Hence, for any $x \in L$ such that $x R y,\left(H\left(f^{-}\right)\right)^{-}(y) \geq f(x)$ and so $\bigwedge\left\{\left(H\left(f^{-}\right)\right)^{-}(y) \mid x R y\right\} \geq f(x)$. Hence, $G^{*}\left(P^{*}(f)\right)(x) \geq f(x)$ and so $G^{*} P^{*}(f) \geq f$, similarly, $H^{*} F^{*}(f) \geq f$. Moreover, for $f \in L_{2}^{X}$ and $x \in X$, by $(B L 14)$, we get that

$$
\begin{aligned}
\left(G^{*}\left(f^{--}\right)(x)\right)^{--} & =\left(\bigwedge\left\{f^{--}(y) \mid y R x\right\}\right)^{--} \\
& =\bigwedge\left\{f^{--}(y) \mid y R x\right\} \\
& =\bigwedge\{f(y) \mid y R x\} \\
& =G^{*}(f)(x) .
\end{aligned}
$$

Hence, $\left(G^{*}\left(f^{--}\right)\right)^{--}=G^{*}(f)$ and similarly we have $\left(H^{*}\left(f^{--}\right)\right)^{--}=$ $H^{*}(f)$. Therefore, $\left(L_{2}^{X} ; G^{*}, H^{*}\right)$ is a tense $B L$-algebra.

Proposition 3.6. In any tense $B L$-algebra $(L ; G, H)$, the following statements hold for any $x, y \in L$ :

(i) If $x \leq y$, then $G(x) \leq G(y), H(x) \leq H(y), F(x) \leq F(y)$ and $P(x) \leq$ $P(y)$.

(ii) $G(x \rightarrow y) \leq F(x) \rightarrow F(y)$ and $H(x \rightarrow y) \leq P(x) \rightarrow P(y)$.

(iii) $x \odot F(y) \leq F(P(x) \odot y)$ and $x \odot P(y) \leq P(F(x) \odot y)$.

(iv) $P \leq P G P$ and $F \leq F H F$.

(v) $P G(x) \leq x^{--}$and $F H(x) \leq x^{--}$.

(vi) $G(x) \odot G(y) \leq G(x \odot y)$ and $H(x) \odot H(y) \leq H(x \odot y)$.

Proof:

(i) If $x \leq y$, for $x, y \in L$, then by $(B L 6), x \rightarrow y=1$. From (TBL0), $G(x \rightarrow y)=H(x \rightarrow y)=1$ and from $(T B L 1), G(x \rightarrow y) \leq G(x) \rightarrow G(y)$ and $H(x \rightarrow y) \leq H(x) \rightarrow H(y)$. Hence, $G(x) \rightarrow G(y)=1$ and $H(x) \rightarrow$ $H(y)=1$. Therefore, $G(x) \leq G(y)$ and $H(x) \leq H(y)$. Moreover, if $x \leq y$, for $x, y \in L$, then by $(B L 8), y^{-} \leq x^{-}$and so $G\left(y^{-}\right) \leq G\left(x^{-}\right)$and $H\left(y^{-}\right) \leq$ 
$H\left(x^{-}\right)$. Hence, by $(B L 8)$, we conclude that $\left(G\left(x^{-}\right)\right)^{-} \leq\left(G\left(y^{-}\right)\right)^{-}$and $\left(H\left(x^{-}\right)\right)^{-} \leq\left(H\left(y^{-}\right)\right)^{-}$and so $F(x) \leq F(y)$ and $P(x) \leq P(y)$.

(ii) Since by (BL8) and (BL12), $x \rightarrow y \leq x \rightarrow y^{--}=x \rightarrow\left(y^{-} \rightarrow\right.$ $0)=y^{-} \rightarrow x^{-}$, so by $(i),(T B L 1)$ and $(B L 9)$, we have

$$
\begin{aligned}
G(x \rightarrow y) & \leq G\left(y^{-} \rightarrow x^{-}\right) \\
& \leq G\left(y^{-}\right) \rightarrow G\left(x^{-}\right) \\
& \leq\left(G\left(x^{-}\right) \rightarrow 0\right) \rightarrow\left(G\left(y^{-}\right) \rightarrow 0\right) \\
& =\left(G\left(x^{-}\right)\right)^{-} \rightarrow\left(G\left(y^{-}\right)\right)^{-} \\
& =F(x) \rightarrow F(y) .
\end{aligned}
$$

The other inequality for $H$, is proved analogously.

(iii) Since $x \odot y \leq x \odot y$, by $(B L 3)$, we get that $x \leq y \rightarrow x \odot y$. Consider $x=P(x)$, so $P(x) \leq y \rightarrow P(x) \odot y$. By $(i)$ and $(i i)$,

$G(P(x)) \leq G(y \rightarrow P(x) \odot y) \leq F(y \rightarrow(P(x) \odot y)) \leq F(y) \rightarrow F(P(x) \odot y)$.

Since by $(T B L 3), x \leq G P(x)$, we get that $x \leq F(y) \rightarrow F(P(x) \odot y)$ and so by $(B L 3), x \odot F(y) \leq F(P(x) \odot y)$. By similar way, $x \odot P(x) \leq P(F(x) \odot y)$.

(iv) From (TBL3), $x \leq G P(x)$ and $x \leq H F(x)$, so by $(i), P(x) \leq$ $P G P(x)$ and $F(x) \leq F H F(x)$. Hence, $P \leq P G P$ and $F \leq F H F$.

(v) From (TBL3), $x^{-} \leq H F\left(x^{-}\right)$, by $(B L 12), x \leq x^{--}$and by $(i)$, $G(x) \leq G\left(x^{--}\right)$. By $(B L 8), G\left(x^{--}\right)^{-} \leq G(x)^{-}$and so by $(i), H F\left(x^{-}\right)=$ $H\left(G\left(x^{--}\right)^{-}\right) \leq H\left(G(x)^{-}\right)$. Hence, $x^{-} \leq H\left(G(x)^{-}\right)$and so by $(B L 8)$, $\left(H\left(G(x)^{-}\right)\right)^{-} \leq x^{--}$. Therefore, $P G(x) \leq x^{--}$. By similar way, $F H(x) \leq$ $x^{--}$.

(v) By $(T B L 1)$ and $(B L 8)$,

$$
\begin{aligned}
G(x \rightarrow y) \odot G(x) & \leq(G(x) \rightarrow G(y)) \odot G(x) \\
& =G(x) \wedge G(y) \\
& \leq G(y)
\end{aligned}
$$

taking $y=x \odot y$, it follows that $G(x \rightarrow x \odot y) \odot G(x) \leq G(x \odot y)$. Since by $(B L 10), y \rightarrow(x \rightarrow x \odot y)=x \odot y \rightarrow x \odot y=1$, we have $y \leq x \rightarrow x \odot y$ and so by $(i), G(y) \leq G(x \rightarrow x \odot y)$. Hence, by $(B L 8)$, 


$$
\begin{aligned}
G(y) \odot G(x) & \leq G(x \rightarrow x \odot y) \odot G(x) \\
& \leq G(x \odot y) .
\end{aligned}
$$

Therefore, $G(x) \odot G(y) \leq G(x \odot y)$. The proof for $H$ is similar.

In the following, we study relation among tense $B L$-algebras, tense $M V$-algebras and tense Boolean algebras.

Theorem 3.7. Let $(L ; G, H)$ be a tense $B L$-algebra and $x^{--}=x, x^{2}=x$, for any $x \in L$. Then $(L ; G, H)$ is a tense $M V$-algebra.

Proof: Let $(L ; G, H)$ be a tense $B L$-algebra and $x^{--}=x, x^{2}=x$, for any $x \in L$. Then by Definition $3.1,(A 0),(A 1)$ and $(A 5)$ are established. We will prove $(A 2),(A 3)$ and $(A 4)$. By $(B L 12), x, y \leq y^{-} \rightarrow x=y \oplus x=$ $x \oplus y$ and by Proposition 3.6(i), $G(x), G(y) \leq G(x \oplus y)$ and so $G(x) \oplus$ $G(y) \leq G(x \oplus y) \oplus G(x \oplus y)$. Since $x^{--}=x, x^{2}=x$, for any $x \in L$, we get that $x \oplus x=\left(x^{-} \odot x^{-}\right)^{-}=\left(x^{-}\right)^{-}=x$, for any $x \in L$. Hence, $G(x) \oplus G(y) \leq G(x \oplus y)$ and by similar way, $H(x) \oplus H(y) \leq H(x \oplus y)$ and so $(A 2)$ is established. Since $x \oplus x=x$, for any $x \in L$, we have $G(x \oplus x)=G(x)=G(x) \oplus G(x), H(x \oplus x)=H(x)=H(x) \oplus H(x)$, $F(x \oplus x)=F(x)=F(x) \oplus F(x)$ and $P(x \oplus x)=P(x)=P(x) \oplus P(x)$. Therefore, $(A 3)$ and $(A 4)$ hold and so $(L ; G, H)$ is a tense $M V$-algebra.

Theorem 3.8. Let $(L ; G, H)$ be a tense BL-algebra and $x^{--}=x, x^{2}=x$, $G\left(x^{-}\right)=G(x)^{-}$and $H\left(x^{-}\right)=H(x)^{-}$for any $x \in L$. Then $(L ; G, H)$ is a tense Boolean algebra.

Proof: Let $(L ; G, H)$ be a tense $B L$-algebra and $x^{--}=x, x^{2}=x$, $G\left(x^{-}\right)=G(x)^{-}$and $H\left(x^{-}\right)=H(x)^{-}$for any $x \in L$. Then by Definition 2.1, $L$ is a Boolean algebra and by Theorem 3.7, $(L ; G, H)$ is a tense $M V$ algebra. By Definition 2.6, (B1) and (B3) hold. Now, we will prove (B2). Since $x \wedge y \leq x, y$, by Proposition 3.6(i), we get that $G(x \wedge y) \leq G(x), G(y)$ and so $G(x \wedge y) \leq G(x) \wedge G(y)$. Now, by Proposition 3.6(vi) and (A2), for $x, y \in L$, we have 


$$
\begin{aligned}
G(x \wedge y) & =G\left(x \odot\left(x^{-} \oplus y\right)\right. \\
& \geq G(x) \odot\left(G\left(x^{-} \oplus y\right)\right) \\
& \geq G(x) \odot\left(G\left(x^{-}\right) \oplus G(y)\right) \\
& \geq G(x) \odot\left(G(x)^{-} \oplus G(y)\right) \\
& \geq G(x) \odot(G(x) \rightarrow G(y)) \\
& \geq G(x) \wedge G(y) .
\end{aligned}
$$

Therefore, $G(x \wedge y)=G(x) \wedge G(y)$, by similar way, we conclude $H(x \wedge y)=$ $H(x) \wedge H(y)$. Moreover, for $x, y \in L$,

$$
\begin{aligned}
G(x \vee y) & =G\left(\left(x^{-} \wedge y^{-}\right)^{-}\right) \\
& =\left(G\left(x^{-} \wedge y^{-}\right)\right)^{-} \\
& =\left(G\left(x^{-}\right) \wedge G\left(y^{-}\right)\right)^{-} \\
& =\left(G(x)^{-} \wedge G(y)^{-}\right)^{-} \\
& =G(x)^{--} \vee G(y)^{--} \\
& =G(x) \vee G(y) .
\end{aligned}
$$

Similarly, we conclude $H(x \vee y)=H(x) \vee H(y)$. Therefore, $(B 2)$ hold and so $(L ; G, H)$ is a tense Boolean algebra.

Definition 3.9. Let $(L ; G, H)$ be a tense $B L$-algebra. Then we define two unary operations $d$ and $\rho$ on $L$ by $d(x)=x \wedge G(x) \wedge H(x)$ and $\rho(x)=x \odot$ $G(x) \odot H(x)$, for any $x \in L$. We observe that for any $x \in L, \rho(x) \leq d(x) \leq x$ and if $(L ; G, H)$ is a tense Boolean algebra, then $\rho(x)=d(x)$. Now, we define $d^{n}(x)$ and $\rho^{n}(x)$, for any $n \in \mathbb{N}$ and for any $x \in L$, by induction as follow:

$$
d^{0}(x)=\rho^{0}(x)=x, d^{n+1} x=d\left(d^{n}(x)\right), \rho^{n+1}(x)=\rho\left(\rho^{n}(x)\right) .
$$

Moreover, for nonempty subset $X$ of $L, \rho^{k}(X)$ is define as follow:

$$
\rho^{0}(X)=X, \rho(X)=\{\rho(x) \mid x \in X\}, \rho^{k+1}(X)=\rho\left(\rho^{k}(X)\right) .
$$

LEMmA 3.10. In any tense $B L$-algebra $(L ; G, H)$, for any $x, y \in L$ and $n \in \mathbb{N}$, the following statements hold:

(i) $d^{n}(0)=0, d^{n}(1)=1, d^{n+1}(x) \leq d^{n}(x)$.

(ii) If $x \leq y$, then $d^{n}(x) \leq d^{n}(y)$. 
(iii) $x=d(x)$ if and only if $d^{n}(x)=x$, for any $n \in \mathbb{N}$.

(iv) $x \leq d^{n}\left(d^{n}\left(x^{-}\right)\right)^{-}$.

(v) If $d(x)=x$, then $d\left(x^{-}\right)=x^{-}$.

\section{ProOF:}

(i) $d(0)=0 \wedge G(0) \wedge H(0)=0$ so $d^{2}(0)=d(d(0))=d(0)=0, \ldots$, $d^{n}(0)=d\left(d^{n-1}(0)\right)=0$ and $d(1)=1 \wedge G(1) \wedge H(1)=1$ so $d^{2}(1)=$ $d(d(1))=d(1)=1, \ldots, d^{n}(1)=d\left(d^{n-1}(1)\right)=d(1)=1$ and $d^{n+1}(x)=$ $d\left(d^{n}(x)\right)=d^{n}(x) \wedge G\left(d^{n}(x)\right) \wedge H\left(d^{n}(x)\right) \leq d^{n}(x)$.

(ii) If $x \leq y$, then by Proposition 3.6(i), $G(x) \leq G(y)$ and $H(x) \leq$ $H(y)$. Therefore,

$$
d(x)=x \wedge G(x) \wedge H(x) \leq y \wedge G(y) \wedge H(y)=d(y)
$$

and so $d(d(x)) \leq d(d(y))$. Hence, $d^{n}(x) \leq d^{n}(y)$.

(iii) If $x=d(x)$, then

$$
\begin{aligned}
& d^{2}(x)=d(d(x))=d(x)=x \\
& d^{3}(x)=d\left(d^{2}(x)\right)=d(x)=x \\
& \vdots \\
& d^{n}(x)=d\left(d^{n-1}(x)\right)=d(x)=x .
\end{aligned}
$$

If $d^{n}(x)=x$, for any $n \in \mathbb{N}$, then for $n=1, d(x)=x$.

(iv) We prove by induction on $n$. If $n=1$, then by (TBL2)

$$
\begin{aligned}
x & \leq x \wedge G P(x) \wedge H F(x) \\
& \leq(x \vee P(x) \vee F(x)) \wedge G(x \vee P(x) \wedge F(x)) \wedge H(x \vee P(x) \vee F(x)) \\
& =d(x \vee P(x) \vee F(x)) \\
& \leq d\left(x^{--} \vee\left(H\left(x^{-}\right)\right)^{-} \vee\left(G\left(x^{-}\right)\right)^{-}\right) \\
& =d\left(\left(x^{-} \wedge H\left(x^{-}\right) \wedge G\left(x^{-}\right)\right)^{-}\right) \\
& =d\left(d\left(x^{-}\right)\right)^{-} .
\end{aligned}
$$

Suppose that the inequality holds for $n$, then we show that it is correct for $n+1$. Since $x \leq d\left(d\left(x^{-}\right)\right)^{-}$, consider $z=\left(d^{n}\left(x^{-}\right)\right)^{-}$, we have: 


$$
\begin{aligned}
\left(d^{n}\left(x^{-}\right)\right)^{-} & =z \\
& \leq d\left(d\left(z^{-}\right)\right)^{-} \\
& =d\left(d\left(d^{n}\left(x^{-}\right)\right)^{--}\right)^{-} \\
& \leq d\left(d\left(d^{n}\left(x^{-}\right)\right)\right)^{-} \\
& =d\left(d^{n+1}\left(x^{-}\right)\right)^{-} .
\end{aligned}
$$

Now by $(i), d^{n}\left(d^{n}\left(x^{-}\right)\right)^{-} \leq d^{n}\left(d\left(d^{n+1}\left(x^{-}\right)\right)^{-}\right)=d^{n+1}\left(d^{n+1}\left(x^{-}\right)\right)^{-}$and since $x \leq d^{n}\left(d^{n}\left(x^{-}\right)\right)^{-}$, so we get that $x \leq d^{n+1}\left(d^{n+1}\left(x^{-}\right)\right)^{-}$. Therefore, (iv) follows by induction.

$(v)$ If $d(x)=x$, then by (iv), $x^{-} \leq d\left(d\left(x^{--}\right)\right)^{-} \leq d(d(x))^{-}=d\left(x^{-}\right)$. Also, $d\left(x^{-}\right)=x^{-} \wedge G\left(x^{-}\right) \wedge H\left(x^{-}\right) \leq x^{-}$and so $d\left(x^{-}\right)=x^{-}$.

Proposition 3.11. In any tense $B L$-algebra $(L ; G, H)$, for any $x, y \in L$ and $k, n \in \mathbb{N}$, the following statements hold:

(i) $\rho^{n}(0)=0, \rho^{n}(1)=1, \rho^{n+1}(x) \leq \rho^{n}(x)$.

(ii) If $x \leq y$, then $\rho^{n}(x) \leq \rho^{n}(y)$.

(iii) $\rho^{k}(x) \odot \rho^{k}(y) \leq \rho^{k}(x \odot y)$.

(iv) $\rho^{k}\left(x^{n}\right) \geq\left(\rho^{k}(x)\right)^{n}$.

ProOF:

(i) $\rho(0)=0 \odot G(0) \odot H(0)=0$ so $\rho^{2}(0)=\rho(\rho(0))=\rho(0)=0, \ldots$, $\rho^{n}(0)=\rho\left(\rho^{n-1}(0)\right)=0$ and $\rho(1)=1 \odot G(1) \odot H(1)=1$ and so $\rho^{2}(1)=$ $\rho(\rho(1))=\rho(1)=1, \ldots, \rho^{n}(1)=\rho\left(\rho^{n-1}(1)\right)=\rho(1)=1$. Moreover, for $x \in L$, $\rho^{n+1}(x)=\rho\left(\rho^{n}(x)\right)=\rho^{n}(x) \odot G\left(\rho^{n}(x)\right) \odot H\left(\rho^{n}(x)\right) \leq \rho^{n}(x)$.

(ii) If $x \leq y$, for $x, y \in L$, then by Proposition 3.6(i), $G(x) \leq G(y)$ and $H(x) \leq H(y)$. Therefore, $\rho(x)=x \odot G(x) \odot H(x) \leq y \odot G(y) \odot H(y)=\rho(y)$, and so $\rho(\rho(x)) \leq \rho(\rho(y))$. Hence, $\rho^{n}(x) \leq \rho^{n}(y)$.

(iii) By Proposition 3.6(vi), for $x, y \in L$ :

$$
\begin{aligned}
\rho(x) \odot \rho(y) & =(x \odot G(x) \odot H(x)) \odot(y \odot G(y) \odot H(y)) \\
& =(x \odot y) \odot(G(x) \odot G(y)) \odot(H(x) \odot H(y)) \\
& \leq x \odot y \odot G(x \odot y) \odot H(x \odot y) \\
& =\rho(x \odot y) .
\end{aligned}
$$


By induction, let $\rho^{n}(x) \odot \rho^{n}(y) \leq \rho^{n}(x \odot y)$, for $x, y \in L$. Then by Proposition 3.6(vi),

$$
\begin{aligned}
\rho^{n+1}(x) \odot \rho^{n+1}(y)= & \rho\left(\rho^{n}(x)\right) \odot \rho\left(\rho^{n}(y)\right) \\
= & \left(\rho^{n}(x) \odot G\left(\rho^{n}(x)\right) \odot H\left(\rho^{n}(x)\right)\right) \\
& \odot\left(\rho^{n}(y) \odot G\left(\rho^{n}(y)\right) \odot H\left(\rho^{n}(y)\right)\right) \\
= & \left(\rho^{n}(x) \odot \rho^{n}(y)\right) \odot\left(G\left(\rho^{n}(x)\right) \odot G\left(\rho^{n}(y)\right)\right) \\
& \odot\left(H\left(\rho^{n}(x) \odot H\left(\rho^{n}(y)\right)\right)\right. \\
\leq & \rho^{n}(x \odot y) \odot G\left(\rho^{n}(x) \odot \rho^{n}(y)\right) \odot H\left(\rho^{n}(x) \odot \rho^{n}(y)\right) \\
\leq & \rho^{n}(x \odot y) \odot G\left(\rho^{n}(x \odot y) \odot H\left(\rho^{n}(x \odot y)\right)\right. \\
= & \rho\left(\rho^{n}(x \odot y)\right) \\
= & \rho^{n+1}(x \odot y) .
\end{aligned}
$$

(iv) By (iii), for $x \in L$, we get that $\left(\rho^{k}(x)\right)^{n}=\rho^{k}(x) \odot \rho^{k}(x) \odot \ldots \odot$ $\rho^{k}(x) \leq \rho^{k}(x \odot x \odot \ldots \odot x)=\rho^{k}\left(x^{n}\right)$.

\section{Tense filters in $B L$-algebras and simple tense $B L$-algebras}

In this section, we introduce the notions of tense filters in $B L$-algebras and simple tense $B L$-algebras and we give some related results.

Definition 4.1. Let $(L ; G, H)$ be a tense $B L$-algebra and $F$ be a filter of $L$. Then $F$ is called a tense filter if $G(x) \in F$ and $H(x) \in F$, for all $x \in F$. Not that if $F$ is a tense filter of tense $B L$-algebra $(L ; G, H)$, then $\rho(x) \in F$ and $d(x) \in F$, for any $x \in F$.

Example 4.2. [10] Let $L=\{0, a, b, 1\}$, where $0<a<b<1$ and $x \wedge y=$ $\min \{x, y\}, x \vee y=\max \{x, y\}$ and operations $\odot$ and $\rightarrow$ are defined as the following tables:

Table 3

\begin{tabular}{|c|c|c|c|c|}
\hline$\odot$ & 0 & $a$ & $b$ & 1 \\
\hline 0 & 0 & 0 & 0 & 0 \\
$a$ & 0 & $a$ & $a$ & $a$ \\
$b$ & 0 & $a$ & $b$ & $b$ \\
1 & 0 & $a$ & $b$ & 1 \\
\hline
\end{tabular}

\section{Table 4}

\begin{tabular}{|l|l|l|l|l|}
\hline$\rightarrow$ & 0 & $a$ & $b$ & 1 \\
\hline 0 & 1 & 1 & 1 & 1 \\
$a$ & 0 & 1 & 1 & 1 \\
$b$ & 0 & $a$ & 1 & 1 \\
1 & 0 & $a$ & $b$ & 1 \\
\hline
\end{tabular}


Then $(L, \vee, \wedge, \odot, \rightarrow, 0,1)$ is a $B L$-algebra and it is not an $M V$-algebra. We define the operations $G=H$ on $L$ as $G(0)=0, G(a)=G(b)=G(1)=1$. It is not difficult to check that $G$ and $H$ are tense operators on $L$. Now, let $F_{1}=\{1\}$ and $F_{2}=\{1, b\}$. Then $F_{1}$ and $F_{2}$ are tense filters of $L$.

Theorem 4.3. The tense filter $[X)$ of tense $B L$-algebra $(L ; G, H)$ generated by nonempty subset $X$ has the following form:

$$
[X)=\left\{y \in L \mid y \geq a_{1} \odot \ldots \odot a_{n}, a_{i} \in \rho^{k_{i}}(X) ; i=1, \ldots, n, k_{i} \in \mathbb{N}, n \geq 1\right\} .
$$

Proof: Let $A=\left\{y \in L \mid y \geq a_{1} \odot \ldots \odot a_{n}, a_{i} \in \rho^{k_{i}}(X) ; i=1, \ldots, n, k_{i} \in\right.$ $\mathbb{N}, n \geq 1\}$. Firstly, we prove that $A$ is a tense filter of $L$. Obviously $1 \in A$. Let $x, y \in A$. Then there exist $a_{1}, \ldots, a_{n}, b_{1}, \ldots, b_{m} \in L$ such that $a_{i} \in \rho^{k_{i}}(X), b_{j} \in \rho^{t_{j}}(X), k_{i}, t_{j} \in \mathbb{N}, m, n \geq 1,1 \leq i \leq n, 1 \leq j \leq m$ and $x \geq a_{1} \odot a_{2} \odot \ldots \odot a_{n}, y \geq b_{1} \odot \ldots \odot b_{m}$. Hence, $x \odot y \geq a_{1} \odot a_{2} \odot \ldots \odot$ $a_{n} \odot b_{1} \odot \ldots \odot b_{m}$ and so $x \odot y \in A$. If $x \leq y$ and $x \in A$, then, there exist $a_{1}, \ldots, a_{p} \in L$ such that $a_{i} \in \rho^{k_{i}}(X)$ and $a_{1} \odot \ldots \odot a_{p} \leq x$, since $x \leq y$, we get that $a_{1} \odot a_{2} \odot \ldots \odot a_{p} \leq y$. Hence, $y \in A$. Thus, $A$ is a filter of $L$. Now, we show that $A$ is a tense filter. If $x \in A$, then there exist $a_{1}, \ldots, a_{w} \in L$, $a_{i} \in \rho^{k_{i}}(X)$ and $a_{1} \odot a_{2} \odot \ldots \odot a_{w} \leq x$. Since $a_{i} \in \rho^{k_{i}}(X)$, by Definition 3.9, there exist $x_{i} \in X$, such that $a_{i}=\rho^{k_{i}}\left(x_{i}\right)$ for any $i(1 \leq i \leq w)$. Hence, $a_{1} \odot a_{2} \odot \ldots \odot a_{w}=\rho^{k_{1}}\left(x_{1}\right) \odot \rho^{k_{2}}\left(x_{2}\right) \odot \ldots \odot \rho^{k_{w}}\left(x_{w}\right) \leq x$, by Proposition $3.11(i i)$, we have

$$
\rho\left(a_{1} \odot \ldots \odot a_{w}\right) \leq \rho(x)=x \odot G(x) \odot H(x) \leq G(x), H(x)
$$

and since by Proposition 3.11(iii),

$$
\rho\left(a_{1}\right) \odot \rho\left(a_{2}\right) \odot \ldots \odot \rho\left(a_{w}\right) \leq \rho\left(a_{1} \odot \ldots \odot a_{w}\right)
$$

we get that $\rho\left(a_{1}\right) \odot \rho\left(a_{2}\right) \odot \ldots \odot \rho\left(a_{w}\right) \leq G(x), H(x)$. Hence, $\rho^{k_{1}+1}\left(x_{1}\right) \odot$ $\rho^{k_{2}+1}\left(x_{2}\right) \odot \ldots \odot \rho^{k_{w}+1}\left(x_{w}\right) \leq G(x), H(x)$ and so $G(x), H(x) \in A$. Therefore, $A$ is a tense filter of $L$. If $x \in X$, since $x \geq \rho(x)$, we conclude $x \in A$. Hence, $X \subseteq A$. Now, let $B$ be a tense filter containing $X$ and $z \in A$, then there exist $a_{1}, \ldots, a_{n} \in L$ such that $a_{i} \in \rho^{k_{i}}(X)$ and $a_{1} \odot a_{2} \odot \ldots \odot a_{n} \leq x$, i.e. $\rho^{k_{i}}\left(x_{1}\right) \odot \ldots \odot \rho^{k_{n}}\left(x_{n}\right) \leq x$. Since $x_{i} \in X \subseteq B$ and $B$ is a tense filter. we get that $\rho^{k_{i}}\left(x_{i}\right) \in B$ and so $\rho^{k_{1}}\left(x_{1}\right) \odot \ldots \odot \rho^{k_{n}}\left(x_{n}\right) \in B$ and since $\rho^{k_{1}}\left(x_{1}\right) \odot \ldots \odot \rho^{k_{n}}\left(x_{n}\right) \leq x$, we have $x \in B$. Therefore, $A$ is a the least tense filter of $L$ containing $X$ and so $[X)=A$. 
Proposition 4.4. Let $(L ; G, H)$ be a tense $B L$-algebra and $x \in L$. Then

$$
[x)=\left\{y \in L \mid y \geq\left(\rho^{k}(x)\right)^{n} ; \text { for some } n, k \in \mathbb{N}\right\} .
$$

Proof: By Theorem 4.3, $[x)=\left\{y \in L \mid y \geq a_{1} \odot a_{2} \odot \ldots \odot a_{n}, a_{i} \in\right.$ $\left.\rho^{k_{i}}(x) ; k_{i} \in \mathbb{N}, 1 \leq i \leq n, n \in \mathbb{N}\right\}$. Consider $k=\max \left\{k_{1}, k_{2}, \ldots, k_{n}\right\}$ such that $a_{i} \in \rho^{k_{i}}(x)$. By Proposition $3.11(i)$, we get that $\rho^{k_{i}}(x) \geq \rho^{k}(x)$. Now, we have

$y \geq \rho^{k_{1}}(x) \odot \rho^{k_{2}}(x) \odot \ldots \odot \rho^{k_{n}}(x) \geq \rho^{k}(x) \odot \rho^{k}(x) \odot \ldots \odot \rho^{k}(x)=\left(\rho^{k}(x)\right)^{n}$.

Hence, $y \geq\left(\rho^{k}(x)\right)^{n}$ and so $[x) \subseteq\left\{y \in L \mid y \geq\left(\rho^{k}(x)\right)^{n} ;\right.$ for some $\left.n, k \in \mathbb{N}\right\}$. If $y \in L$, such that $y \geq\left(\rho^{k}(x)\right)^{n}$, then $y \geq \rho^{k}(x) \odot \rho^{k}(x) \odot \ldots \odot \rho^{k}(x)$ and so by Theorem 4.3, $y \in[x)$. Therefore,

$$
[x)=\left\{y \in L \mid y \geq\left(\rho^{k}(x)\right)^{n} ; \text { for some } n, k \in \mathbb{N}\right\} .
$$

Proposition 4.5. Let $F$ be a tense filter of tense $B L$-algebra $(L ; G, H)$ and $x \in L$. Then the tense filter generated by $F \cup\{x\}$ is characterized as

$$
[F \cup\{x\})=\left\{y \in L \mid y \geq a \odot\left(\rho^{k}(x)\right)^{n} ; \text { for some } a \in F, k, n \in \mathbb{N}\right\}
$$

Proof: Let $A=\left\{y \in L \mid y \geq a \odot\left(\rho^{k}(x)\right)^{n} ;\right.$ for some $\left.a \in F, k, n \in \mathbb{N}\right\}$. We prove that $A$ is the least tense filter of $L$ containing $F \cup\{x\}$. Let $x, y \in A$, then there exist $a, b \in F, k, k^{\prime}, n, n^{\prime} \in \mathbb{N}$ such that $x \geq a \odot\left(\rho^{k}(x)\right)^{n}$ and $y \geq b \odot\left(\rho^{k^{\prime}}(x)\right)^{n^{\prime}}$.

Hence, $x \odot y \geq\left(a \odot\left(\rho^{k}(x)\right)^{n}\right) \odot b \odot\left(\rho^{k^{\prime}}(x)\right)^{n^{\prime}}=(a \odot b) \odot\left(\rho^{k}(x)\right)^{n} \odot\left(\rho^{k^{\prime}}(x)\right)^{n^{\prime}}$. Taking $t=\operatorname{Max}\left\{k, k^{\prime}\right\}$, then by Proposition 3.11(i), $\rho^{k}(x) \geq \rho^{t}(x)$ and $\rho^{k^{\prime}}(x) \geq \rho^{t}(x)$ and so $\left(\rho^{k}(x)\right)^{n} \odot\left(\rho^{k^{\prime}}(x)\right)^{n^{\prime}} \geq\left(\rho^{t}(x)\right)^{n+n^{\prime}}$ and so $x \odot y \geq$ $(a \odot b) \odot\left(\rho^{t}(x)\right)^{n+n^{\prime}}$. Therefore, $x \odot y \in A$. If $x \leq y$ and $x \in A$, then there exist $a \in F$ and $k, n \in \mathbb{N}$ such that $x \geq a \odot\left(\rho^{k}(x)\right)^{n}$. Hence, $y \geq a \odot\left(\rho^{k}(x)\right)^{n}$ and so $y \in A$. Therefore, $A$ is a filter of $L$. If $x \in A$, then there exist $a \in F$ and $k, n \in \mathbb{N} x \geq a \odot\left(\rho^{k}(x)\right)^{n}$, and so by Proposition 3.11(ii), $\rho(x) \geq \rho\left(a \odot\left(\rho^{k}(x)\right)^{n}\right)$. From Proposition 3.11(iii), we get that

$$
\begin{aligned}
\rho(x) \geq \rho\left(a \odot\left(\rho^{k}(x)\right)^{n}\right) & \geq \rho(a) \odot \rho\left(\left(\rho^{k}(x)^{n}\right)\right. \\
& \geq \rho(a) \odot\left(\rho\left(\rho^{k}(x)\right)\right)^{n} \\
& =\rho(a) \odot\left(\rho^{k+1}(x)\right)^{n}
\end{aligned}
$$

and since $F$ is a tense filter of $L$, we get that $\rho(a) \in F$ and since $G(x) \geq$ $\rho(x)$, we have $G(x) \geq \rho(a) \odot\left(\rho^{k+1}(x)\right)^{n}$. Hence, $G(x) \in A$ and similarly, 
$H(x) \in A$. Therefore, $A$ is a tense filter of $L$. Now, if $B$ is a tense filter containing $F \cup\{x\}$ and $z \in A$, then there exist $a \in F$ and $k, n \in \mathbb{N}$ such that, $z \geq a \odot\left(\rho^{k}(x)\right)^{n}$. Since $x \in B$ and $B$ is a tense filter we have $\left(\left(\rho^{k}(x)\right)^{n} \in B\right.$ and since $a \in F \subseteq B$, we get that $a \odot\left(\rho^{k}(x)\right)^{n} \in B$. Hence, $z \in B$ and so $A$ is the least tense filter of $L$ containing $F \cup\{x\}$. Thus,

$$
[F \cup\{x\})=\left\{y \in L \mid y \geq a \odot\left(\rho^{k}(x)\right)^{n} ; \text { for some } a \in F, k, n \in \mathbb{N}\right\} .
$$

As usual, for two filters $F_{1}$ and $F_{2}$ of $B L$-algebra $L$, we let $F_{1} \wedge F_{2}:=$ $F_{1} \cap F_{2}$ and $F_{1} \vee F_{2}=\left[F_{1} \cup F_{2}\right)$ and it is easy to check

$$
F_{1} \vee F_{2}=\left\{y \mid y \geq x_{1} \odot x_{2} ; \text { for some } x_{1} \in F_{1}, x_{2} \in F_{2}\right\}
$$

ThEOREM 4.6. $F_{t}(L)$ of all tense filter of tense $B L$-algebra $(L ; G, H)$ is a complete sublattice of $F(L)$ of all filter of $L$.

Proof: Let $F_{1}$ and $F_{2}$ be two tense filter and $x \in F_{1} \wedge F_{2}$. Then $x \in F_{1}$ and $x \in F_{2}$ so $G(x) \in F_{1}$ and $G(x) \in F_{2}$. Hence, $G(x) \in F_{1} \wedge F_{2}$ and by similar way $H(x) \in F_{1} \wedge F_{2}$. Also, if $x \in F_{1} \vee F_{2}$, then there exist $x_{1} \in F_{1}$ and $x_{2} \in F_{2}$ such that $x \geq x_{1} \odot x_{2}$. Now by Proposition 3.6(i) and $(v i)$, we get that $G(x) \geq G\left(x_{1} \odot x_{2}\right) \geq G\left(x_{1}\right) \odot G(x)$. Since $F_{1}$ and $F_{2}$ are tense filters, we conclude that $G\left(x_{1}\right) \in F_{1}$ and $G\left(x_{2}\right) \in F_{2}$ and so $G(x) \in\left[F_{1} \cup F_{2}\right)=F_{1} \vee F_{2}$. By similar way, $H(x) \in F_{1} \vee F_{2}$. Therefore, $F_{1} \vee F_{2}$ is a tense filter and so $F_{t}(L)$ is complete sublattice of $F(L)$.

ThEOREM 4.7. Let $F$ be a proper tense filter of tense $B L$-algebra $(L ; G, H)$. Then the following statements are equivalent:

(i) $F$ is a maximal tense filter of $(L ; G, H)$,

(ii) for each $x \in L \backslash F$, there exist $a \in F$ and $k, m \in \mathbb{N}$ such that $a \odot$ $\left(\rho^{k}(x)\right)^{m}=0$.

\section{ProOF:}

$(i) \Rightarrow($ ii $)$ Let $F$ be a maximal tense filter of tense $B L$-algebra $(L ; G, H)$ and $x \in L \backslash F$. Then by $F \subset[F \cup\{x\}) \subseteq L$, we conclude that $[F \cup\{x\})=L$ and since $0 \in L$, we get that $0 \in[F \cup\{x\})$. From Proposition 4.5, there exist $a \in F$ and $k, m \in \mathbb{N}$ such that $0 \geq a \odot\left(\left(\rho^{k}(x)\right)^{m}\right.$ and so $a \odot\left(\rho^{k}(x)\right)^{m}=0$.

$($ ii $) \Rightarrow($ i) Let $E$ be a tense filter of $L$ such that $F \subset E \subseteq L$. If there exist $x \in E \backslash F$, then by (ii) there exist $b \in F$ and $k, m \in \mathbb{N}$ such that 
$b \odot\left(\rho^{k}(x)\right)^{m}=0$. Now, Since $b \in F \subseteq E, x \in E$ and $E$ is a tense filter, we get that $\left(\rho^{k}(x)\right)^{m} \in E$ and so $0=b \odot\left(\rho^{k}(x)\right)^{m} \in E$. Hence, $E=L$ and so $F$ is a maximal tense filter of $L$.

TheOrem 4.8. For any tense BL-algebra $(L ; G, H)$, the following statements are equivalent:

(i) $(L ; G, H)$ is a simple tense BL-algebra,

(ii) for any $x \in L \backslash\{1\}$, there exist $k, n \in \mathbb{N}$, such that $\left(\rho^{k}(x)\right)^{n}=0$.

\section{Proof:}

$(i) \Rightarrow(i i)$ Let $(L ; G, H)$ be a simple tense $B L$-algebra. Then $\{1\}$ is a maximal filter of $L$ and so by Theorem 4.7 for any $x \in L \backslash\{1\}$, there exist $k, n \in \mathbb{N}$ such that $1 \odot\left(\rho^{k}(x)\right)^{n}=0$. Therefore, $\left(\rho^{k}(x)\right)^{n}=0$.

$(i i) \Rightarrow(i)$ If for any $x \in L \backslash\{1\}$ there exist $k, n \in \mathbb{N}$ such that $\left(\rho^{k}(x)\right)^{n}=$ 0 , then by Theorem $4.7, F=\{1\}$ is a maximal tense filter and so there is not nontrivial tense filter of $L$ and so $L$ is a simple tense $B L$-algebra.

TheOREM 4.9. Let $F$ be a proper tens filter of tense $B L$-algebra $(L ; G, H)$. Then the following statements are equivalent:

(i) $F$ is a maximal tense filter of $(L ; G, H)$,

(ii) for each $x \in L, x \notin F$ if and only if $\left(\left(\rho^{k}(x)\right)^{n}\right)^{-} \in F$, for some $k, n \in \mathbb{N}$.

\section{ProOF:}

$(i) \Rightarrow($ ii $)$ Let $F$ be a maximal tense filter of $(L ; G, H)$ and $x \in L \backslash F$. Then by Theorem 4.7, there exist $a \in F$ and $n, k \in \mathbb{N}$, such that $a \odot$ $\left(\rho^{k}(x)\right)^{n}=0$. By $(B L 11), a \leq\left(\left(\rho^{k}(x)^{n}\right)^{-}\right.$and since $a \in F$, we conclude that $\left(\left(\rho^{k}(x)^{n}\right)\right)^{-} \in F$. Conversely, let $\left(\left(\rho^{k}(x)\right)^{n}\right)^{-} \in F$ for some $k, n \in \mathbb{N}$. If $x \in F$, then $\rho(x) \in F$ and so $\left(\rho^{k}(x)\right)^{n} \in F$. By $(B L 12), 0=\left(\rho^{k}(x)\right)^{n} \odot$ $\left(\left(\rho^{k}(x)\right)^{n}\right)^{-} \in F$ and so $F=L$ which is contradiction. Therefore, $x \notin F$.

(ii) $\Rightarrow(i)$ Let $F \subset E \subseteq L$ and $E$ be a tense filter of $L$. Then there exists $x \in E$ such that $x \notin F$. By (ii) there exist $k, n \in \mathbb{N}$, such that $\left(\left(\rho^{k}(x)\right)^{n}\right)^{-} \in F \subseteq E$, since $E$ is a tense filter and $x \in E$, we have $\left(\rho^{k}(x)\right)^{n} \in E$ and so by $(B L 12), 0=\left(\rho^{k}(x)\right)^{n} \odot\left(\left(\rho^{k}(x)^{n}\right)\right)^{-} \in E$. Hence $E=L$ and so $F$ is a maximal tense filter of $(L ; G, H)$. 


\section{Tense congruence relations in tense $B L$-algebras}

In this section, we introduce the notions of tense congruence in tense $B L$ algebras and strict tense $B L$-algebras and we give some related results.

DEFinition 5.1. Let $\theta$ be a congruence relation on $B L$-algebra $L$ and $(L ; G, H)$ be a tense $B L$-algebra. Then $\theta$ is called a tense congruence if it is compatible with respect to the operations $G$ and $H$. In fact, if $x \theta y$, then $G(x) \theta G(y)$ and $H(x) \theta H(y)$, for any $x, y \in L$.

Proposition 5.2. Let $(L ; G, H)$ be a tense $B L$-algebra, $F$ be a filter of $L$ and $\theta_{F}$ be a congruence relation induced by $F$. Then $F$ is a tense filter of $L$ if and only if $\theta_{F}$ is a tense congruence.

Proof: Let $\theta_{F}$ be a tense congruence relation induced by $F$ and $x \in F$. Then $1 \rightarrow x \in F$ and $x \rightarrow 1 \in F$ and so $1 \theta_{F} x$. Since $\theta_{F}$ is tense congruence, we get that $G(1) \theta_{F} G(x)$ and $H(1) \theta_{F} H(x)$ and so $1 \theta G(x)$ and $1 \theta H(x)$. Hence, $G(x) \in F$ and $H(x) \in F$ and so $F$ is a tense filter of $L$. Conversely, let $F$ be a tense filter of $L$ and $x \theta_{F} y$, for $x, y \in L$. Then $x \rightarrow y \in F$ and $y \rightarrow x \in F$ and since $F$ is a tense filter of $L$, we have $G(x \rightarrow y) \in F$ and $H(x \rightarrow y) \in F$ and by $(T B L 1), G(x \rightarrow y) \leq G(x) \rightarrow G(y)$ and $H(x \rightarrow y) \leq H(x) \rightarrow H(y)$. Now, since $F$ is a filter of $L$, we conclude that $G(x) \rightarrow G(y) \in F$ and $H(x) \rightarrow H(y) \in F$. By similar way, we get that $G(y) \rightarrow G(x) \in F$ and $H(y) \rightarrow H(x) \in F$. Hence, $G(x) \theta_{F} G(y)$ and $H(x) \theta_{F} H(y)$. Therefore, $\theta_{F}$ is a tense congruence relation on $L$.

Proposition 5.3. Let $(L ; G, H)$ be a tense $B L$-algebra. Then there is an one-to-one correspondence between tense filters of $L$ and tense congruences relations induced by tense filters of $L$.

Proof: It follows by Theorem 2.4 and Proposition 5.2.

Theorem 5.4. Let $(L ; G, H)$ be a tense BL-algebra and $F$ be a filter of $L$. Then $F$ is a tense filter of $L$ if and only if $\left(\frac{L}{F} ; G^{*}, H^{*}\right)$ by the operators $G^{*}, H^{*}: \frac{L}{F} \rightarrow \frac{L}{F}$ such that

$$
G^{*}([x]):=[G(x)], H^{*}([x]):=[H(x)]
$$

and $F^{*}([x]):=[F(x)], P^{*}([x]):=[P(x)]$ is a tense BL-algebra. 
Proof: Let $(L ; G, H)$ be a tense $B L$-algebra and $F$ be a tense filter of $L$. Then by Theorem $2.4,\left(\frac{L}{F}, \cdot, \rightarrow, \sqcup, \sqcap,[0],[1]\right)$ is a $B L$-algebra. Define operators $G^{*}, H^{*}: \frac{L}{F} \rightarrow \frac{L}{F}$ by

$$
G^{*}([x]):=[G(x)], H^{*}([x]):=[H(x)] .
$$

Now, we prove $\left(\frac{L}{F} ; G^{*}, H^{*}\right)$ is a tense $B L$-algebra. Firstly, we prove that operations $G^{*}$ and $H^{*}$ are well-defined. Let $[x]=[y]$. Then $x \rightarrow y, y \rightarrow$ $x \in F$. Since $F$ is a tense filter of $L$, by similar proof of Proposition 5.2, we get that $G(x) \rightarrow G(y) \in F$ and $G(y) \rightarrow G(x) \in F$. Hence, $[G(x)]=[G(y)]$ and so $G^{*}([x])=G^{*}([y])$. Similarly, we have $H^{*}([x])=H^{*}([y])$ and so operations $G^{*}$ and $H^{*}$ are well-defined. By $(T B L 0)$ in tense $B L$-algebra $L, G^{*}([1])=[G(1)]=[1]$ and similarly, $H^{*}([1])=[H(1)]=[1]$, and so $(T B L 0)$ holds in $\frac{L}{F}$. Let $[x],[y] \in \frac{L}{F}$. Then by $(T B L 1)$ in tense $B L$ algebra $L$,

$$
\begin{aligned}
G^{*}([x] \rightarrow[y]) & =G^{*}([x \rightarrow y]) \\
& =[G(x \rightarrow y)] \\
& \leq[G(x) \rightarrow G(y)] \\
& =[G(x)] \rightarrow[G(y)] \\
& \leq G^{*}([x]) \rightarrow G^{*}([y]) .
\end{aligned}
$$

Similarly, we get that $H^{*}([x] \rightarrow[y]) \leq H^{*}([x]) \rightarrow H^{*}([y])$ and so $(T B L 1)$ holds in $\frac{L}{F}$. Finally, By $(T B L 3)$ in tense $B L$-algebra $L$, we have

$$
\begin{aligned}
G^{*} P^{*}([x]) & =G^{*}\left(P^{*}[x]\right) \\
& =G^{*}\left(\left(H^{*}\left[x^{-}\right]\right)^{-}\right) \\
& =G^{*}\left(\left[H\left(x^{-}\right)\right]^{-}\right) \\
& =G^{*}\left(\left[\left(H\left(x^{-}\right)\right)^{-}\right]\right) \\
& =\left[G\left(\left(H\left(x^{-}\right)\right)^{-}\right]\right. \\
& =[G P(x))] \\
& \geq[x] .
\end{aligned}
$$

Similarly, we get that $H^{*} F^{*}([x]) \geq[x]$ and so $(T B L 2)$ holds in $\frac{L}{F}$. More- 
over, for $[x] \in \frac{L}{F}$,

$$
\begin{aligned}
\left(G^{*}\left([x]^{--}\right)\right)^{--} & =\left(G^{*}\left(\left[x^{--}\right]\right)\right)^{--} \\
& =\left(\left[G\left(x^{--}\right)\right]\right)^{--} \\
& =\left[G\left(x^{--}\right)^{--}\right] \\
& =[G(x)] \\
& =G^{*}([x])
\end{aligned}
$$

Similarly, $\left(H^{*}\left([x]^{--}\right)\right)^{--}=H^{*}([x])$. Therefore, $\left(\frac{L}{F} ; G^{*}, H^{*}\right)$ is a tense $B L$-algebra. Conversely, let $F$ be filter of $L, x \in F$ and $\left(\frac{L}{F} ; G^{*}, H^{*}\right)$ is a tense $B L$-algebra. Then $[x]=[1]$ and so $G^{*}([x])=G^{*}([1])$. Hence, $[G(x)]=[1]$ and so $G(x) \in F$. Similarly, $H(x) \in F$ and so $F$ is a tense filter of $L$.

Definition 5.5. Let $\left(L_{1} ; G_{1}, H_{1}\right)$ and $\left(L_{2} ; G_{2}, H_{2}\right)$ be two tense $B L$-algebras and $\phi: L_{1} \rightarrow L_{2}$ be a $B L$-homomorphism. Then $\phi$ is called a tense $B L$-homomorphism (or briefly, a $T B L$-homomorphism) if $G(\phi(x))=$ $\phi(G(x))$ and $H(\phi(x))=\phi(H(x))$, for all $x \in L_{1}$.

Proposition 5.6. Let $\phi:\left(L_{1} ; G_{1}, H_{1}\right) \rightarrow\left(L_{2} ; G_{2}, H_{2}\right)$ be a $T B L$-homomorphism. Then the following statements hold:

(i) $\operatorname{ker} \phi$ is a tense filter of $L_{1}$.

(ii) If $F$ is a tense filter of $L_{2}$, then $\phi^{-1}(F)$ is a tense filter of $L_{1}$.

(iii) If $\operatorname{ker} \phi \subseteq F, \phi$ is onto and $F$ is a tense filter of $L_{1}$, then $\phi(F)$ is a tense filter of $L_{2}$.

Proof:

( $i$ ) It is easy to check that $\operatorname{ker} \phi$ is a filter of $L_{1}$. Now, let $x \in \operatorname{ker} \phi$. Then $\phi(x)=1$ and so $1=G(1)=G(\phi(x))=\phi(G(x))$. Hence, $G(x) \in$ ker $\phi$, by similar way, $H(x) \in \operatorname{ker} \phi$ and so $\operatorname{ker} \phi$ is a tense filter of $L_{1}$.

(ii) Let $F$ be a tense filter of $L_{2}$ and $x \in \phi^{-1}(F)$. Then $\phi^{-1}(F)$ is a filter of $L_{1}$ and $\phi(x) \in F$ and so $\phi(G(x))=G(\phi(x)) \in F$. Hence $G(x) \in \phi^{-1}(F)$, by similar way, $H(x) \in \phi^{-1}(F)$. Therefore, $\phi^{-1}(F)$ is a tense filter of $L_{1}$.

(iii) Assume that $\operatorname{ker} \phi \subseteq F, \phi$ is onto and $F$ is a tense filter of $L_{1}$. Firstly, we prove $\phi(F)$ is a filter of $L_{2}$. Let $a, b \in \phi(F)$. Then there exist 
$x, y \in F$, such that $a=\phi(x), b=\phi(y)$ and $a \odot b=\phi(x) \odot \phi(y)=\phi(x \odot y)$. Since $x \odot y \in F$, we get that $a \odot b \in \phi(F)$. Moreover, if $a \leq b$ and $a \in \phi(F)$, then there exists $z \in F$ and $w \in L_{1}$, such that $a=\phi(z), b=\phi(w)$. Hence, $\phi(z) \leq \phi(w)$ and so $\phi(z \rightarrow w)=1$. Thus, $z \rightarrow w \in \operatorname{ker} \phi \subseteq F$ and since $z \in F$, we get that $w \in F$. Therefore, $b=\phi(w) \in \phi(F)$ and so $\phi(F)$ is a filter of $L_{2}$. Now, let $x \in \phi(F)$. Then there exists $t \in F$, such that $x=\phi(t)$ and since $F$ is a tense filter of $L_{1}$, we have $G(t) \in F$ and so $G(x)=G(\phi(t))=\phi(G(t)) \in \phi(F)$. By similar way, $H(x) \in \phi(F)$ and so $\phi(F)$ is a tense filter of $L_{2}$.

Definition 5.7. A tense $B L$-algebra $(L ; G, H)$ is called strict if for all $x \in L, G(x \odot x)=G(x) \odot G(x)$ and $H(x \odot x)=H(x) \odot H(x)$.

Example 5.8. Let $(L ; G, H)$ be tense $B L$-algebra Example 3.2. Then $(L ; G, H)$ is a strict tense $B L$-algebra.

Proposition 5.9. Let $(L ; G, H)$ be a strict tense $B L$-algebra and $F$ be a tense filter of $L$. Then $\left(\frac{L}{F} ; G^{*}, H^{*}\right)$ is a strict tense $B L$-algebra.

Proof: By Theorem $5.4,\left(\frac{L}{F} ; G^{*}, H^{*}\right)$ is a tense $B L$-algebra, when $F$ is a tense filter of $L$. Let $[x],[y] \in \frac{L}{F}$. Since $(L ; G, H)$ is a strict tense $B L$ algebra, we conclude that

$$
\begin{aligned}
G^{*}([x] \cdot[y]) & =G^{*}([x \odot y]) \\
& =[G(x \odot y)] \\
& =[G(x) \odot G(y)] \\
& =[G(x)] \cdot[G(y)] \\
& =G^{*}([x]) \cdot G^{*}([y]) .
\end{aligned}
$$

Similarly, $H^{*}([x] \cdot[y])=H^{*}([x]) \cdot H^{*}([y])$. Therefore, $\left(\frac{L}{F} ; G^{*}, H^{*}\right)$ is a strict tense $B L$-algebra.

TheOrem 5.10. Let $(L ; G, H)$ be a strict tense BL-algebra and for any $x \in$ $L, x^{--}=x, G\left(x^{-}\right)=(G(x))^{-}$and $H\left(x^{-}\right)=(H(x))^{-}$. Then $(L ; G, H)$ is a tense $M V$-algebra.

Proof: Let $(L ; G, H)$ be a strict tense $B L$-algebra and $x^{--}=x$, for any $x \in L$. Then $L$ is a $M V$-algebra and by Definition 3.1, $(A 0),(A 1)$ and 
$(A 5)$ are hold. Now, we prove $(A 2),(A 3)$ and $(A 4)$. Let $x, y \in L$. Then by Definition 2.5,

$$
\begin{aligned}
G(x) \oplus G(y) & =\left(G(x)^{-} \odot G(y)\right)^{-} \\
& =\left(G\left(x^{-}\right) \odot G\left(y^{-}\right)\right)^{-} \\
& =\left(G\left(x^{-} \odot y^{-}\right)\right)^{-} \\
& =G\left(\left(x^{-} \odot y^{-}\right)^{-}\right) \\
& =G(x \oplus y) .
\end{aligned}
$$

Similarly, $H(x) \oplus H(y)=H(x \oplus y)$ and so $(A 2)$ holds. Moreover, if $y=x$, then $G(x) \oplus G(x)=G(x \oplus x)$ and $H(x) \oplus H(x)=H(x \oplus x)$ and so $(A 3)$ holds. For $(A 4)$, since $(L ; G, H)$ is a strict tense $B L$-algebra, we have

$$
\begin{aligned}
F(x) \oplus F(x) & =G\left(x^{-}\right)^{-} \oplus G\left(x^{-}\right)^{-} \\
& =\left(G\left(x^{-}\right) \odot G\left(x^{-}\right)\right)^{-} \\
& =\left(G\left(x^{-} \odot x^{-}\right)\right)^{-} \\
& =\left(G\left((x \oplus x)^{-}\right)\right)^{-} \\
& =F(x \oplus x) .
\end{aligned}
$$

Similarly, $P(x) \oplus P(y)=P(x \oplus y)$ and so $(A 4)$ holds. Therefore, $(L ; G, H)$ is a tense $M V$-algebra.

\section{Conclusion}

The results of this paper will be devoted to study the notion of the tense operators on $B L$-algebras. We presented a characterization and several important properties of the tense operators on $B L$-algebras. Moreover, we investigated the relation among tense $B L$-algebras, tense $M V$-algebras and tense Boolean algebras. Also, we defined the notions of tense filters and maximal tense filters in $B L$-algebras and we stated and proved some theorems which determine the relationship between this notions and simple tense $B L$-algebra and we proved that the set of all tense filters of a $B L$ algebra is complete sublattice of $F(L)$. Finally, we introduced the notions of tense congruence relations in tense $B L$-algebras and strict tense $B L$ algebras and we shown that there is an one-to-one correspondence between tense filters and tense congruences relations induced by tense filters. 


\section{References}

[1] M. Botur, I. Chajda, R. Halaš, M. Kolařík, Tense operators on basic algebras, International Journal of Theoretical Physics, vol. 50 (2011), pp. 37373749, DOI: https://doi.org/10.1007/s10773-011-0748-4.

[2] J. P. Burgess, Basic Tense Logic, [in:] D. M. Gabbay, F. Guenthner (eds.), Handbook of Philosophical Logic, Springer Netherlands, Dordrecht (2002), pp. 1-42, DOI: https://doi.org/10.1007/978-94-017-0462-5_1.

[3] I. Chajda, Algebraic axiomatization of tense intuitionistic logic, Open Mathematics, vol. 9(5) (2011), pp. 1185-1191, URL: http://eudml.org/ doc/269762.

[4] I. Chajda, M. Kolak, Dynamic effect algebras, Mathematica Slovaca, vol. 62(3) (2012), pp. 379-388, DOI: https://doi.org/10.2478/s12175-0120015-z.

[5] D. Diaconescu, G. Georgescu, Tense Operators on MV-Algebras and Lukasiewicz-Moisil Algebras, Fundamenta Informaticae, vol. 81(4) (2007), pp. 379-408.

[6] A. V. Figallo, G. Gallardo, G. Pelaitay, Tense operators on m-symmetric algebras, International Mathematical Forum, vol. 41 (2011), pp. 20072014.

[7] A. V. Figallo, G. Pelaitay, Tense operators on SHn-algebras, Pioneer Journal Algebra Number Theory and its Applications, vol. 1 (2011), pp. 33-41.

[8] P. Hájek, Metamathematics of fuzzy logic, Springer Netherlands, Dordrecht (1988), DOI: https://doi.org/10.1007/978-94-011-5300-3.

[9] T. Kowalski, Varieties of tense algebras, Reports on Mathematical Logic, vol. 32 (1998), pp. 53-95.

[10] C. Lele, J. B. Nganou, MV-algebras derived from ideals in BL-algebras, Fuzzy Sets and Systems, vol. 218 (2013), pp. 103-113, DOI: https: //doi.org/10.1016/j.fss.2012.09.014.

[11] A. D. Nola, G. Georgescu, A. Iorgulescu, Pseudo BL-algebras: Part I, Multiple-Valued Logic, vol. 8(5-6) (2000), pp. 673-714.

[12] A. D. Nola, L. Leuştean, Compact representations of BL-algebras, Archive for Mathematical Logic, vol. 42 (2003), pp. 737-761, DOI: https://doi. org/10.1007/s00153-003-0178-y. 
Akbar Paad
University of Bojnord
Department of Mathematics
Bojnord, Iran
e-mail: akbar.paad@gmail.com 